\title{
Changes in the use of diabetes drugs among community-dwelling people with Alzheimer's disease
}

\author{
Carlos López-Rubio ${ }^{1 \dagger}$, Marjaana Koponen ${ }^{2,3,4 \dagger}$, Pasi Lampelaa ${ }^{2,3}$, Heidi Taipale ${ }^{3,5,6}$, Antti Tanskanen6, \\ J. Simon Bell ${ }^{3,4}$, Anna-Maija Tolppanen ${ }^{2,3}$ and Sirpa Hartikainen ${ }^{2,3^{*}}$
}

\begin{abstract}
Background: Type 2 diabetes is common in persons with Alzheimer's disease (AD). Management of diabetes in persons with $A D$ is challenging due to changing goals of care and susceptibility to adverse drug events including hypoglycemia. The aim of this study was to investigate the prevalence of diabetes drug use from 5 years before to 5 years after the time of $A D$ diagnosis among persons with and without $A D$.

Methods: This was a nationwide register-based study of persons with and without AD and diabetes in Finland. We analyzed data from the Medication Use and Alzheimer's disease (MEDALZ) study that included 70,718 communitydwelling people diagnosed with AD from 2005 to 2011. The study population included 8418 persons with AD and 6666 matched persons without AD who were diagnosed with diabetes 5 years before AD diagnosis (index date). We defined the prevalence of diabetes drug use in three-month evaluation periods from 5 years before until 5 years after the index date.

Results: Nearly all people with diabetes (94\% in both cohorts) used one or more diabetes drugs on the index date. The most prevalent drug metformin was used by $60.9 \%$ of people with $A D$ and $59.1 \%$ of people without AD. The next most prevalent drugs were sulfonylureas and insulin. The prevalence of diabetes drug use was similar in people with and without AD but began to decline 1 year after AD diagnosis in the AD cohort compared to non-AD cohort.

Conclusions: The decline in diabetes drug use after AD diagnosis may be attributed to clinicians and patients seeking to avoid serious adverse drug events including hypoglycemia. In addition, the findings may reflect personalized glycemic control and unintentional weight loss in persons with AD reducing the need for diabetes drugs.
\end{abstract}

\section{Introduction}

Alzheimer's disease (AD) is a neurodegenerative disease and most common form of dementia that predominately affects older people [1]. The global prevalence of type 2 diabetes has increased in recent decades and 422 million

*Correspondence: sirpa.hartikainen@uef.fi

${ }^{\dagger}$ Carlos López-Rubio and Marjaana Koponen contributed equally to this work.

${ }^{3}$ School of Pharmacy, University of Eastern Finland, PO Box 1627, 70210 Kuopio, Finland

Full list of author information is available at the end of the article people now live with type 1 or type 2 diabetes [2]. Nearly $30 \%$ of persons with diabetes were aged 65 years and older in 2019 [3]. About one quarter (26.8\%) of Americans aged 65 years and older have type 2 diabetes [4]. Both AD and diabetes share several risk factors [5] and their coexistence is common [6]. The benefits and risks of intensive glycemic control in persons with $\mathrm{AD}$ may be different to that of the general population.

Management of diabetes and self-care is challenging for persons with $\mathrm{AD}$ and their caregivers. Persons with $\mathrm{AD}$ may forget to eat meals, experience challenges taking 
medication, and have difficulties in recognizing symptoms of hypoglycemia [7]. Clinical practice guidelines for managing diabetes in persons with cognitive impairment emphasize the importance of an individualized approach focused on maintaining quality of life through preventing hypoglycemia and reducing hyperglycemia [8]. This includes providing care across a continuum with less stringent $\mathrm{HbA1c}$ lowering for people with advanced dementia or who are frail. Deintensification of diabetes treatment regimens may include discontinuing drugs most likely to cause hypoglycemia (e.g. sulfonylureas, insulin) or switching to agents associated with a lower risk of hypoglycemia [9].

However, there is a lack of data on the use of diabetes drugs after $\mathrm{AD}$ diagnosis. One prospective study of persons with type 2 diabetes and dementia reported the total number of diabetes drugs but did not analyze drug groups individually [10]. To our knowledge, no previous studies have investigated changes in the use of diabetes drugs among people with dementia. The aim of this study was to investigate the temporal changes in prevalence of diabetes drugs in relation to $\mathrm{AD}$ diagnosis, and compare the changes in prevalence to a comparison cohort of persons without $\mathrm{AD}$.

\section{Methods}

\section{Study cohort}

We analyzed data from the register-based MEDALZ (Medication Use and Alzheimer's disease) cohort that included 70,718 community-dwelling residents of Finland who received a verified diagnosis of AD from 2005 to 2011 [11]. Each person with $\mathrm{AD}$ was matched to a comparison person without AD according to age-, sexand region of residence $(n=70,718)$. Persons with AD were identified from the Special Reimbursement Register maintained by the Social Insurance Institution of Finland (SII). AD was diagnosed according to NINCDSADRDA [12] and DSM-IV criteria. For a diagnosis of AD to be verified by the SII a person needed to fulfil all the following criteria: (i) symptoms consistent with AD; (ii) a decrease in social capacity over a period of at least 3 months; (iii) received a computed tomography/magnetic resonance imaging scan; (iv) had possible alternative diagnoses excluded; and (v) received confirmation of the diagnosis by a registered geriatrician or neurologist. Additional data were obtained from the Finnish nationwide registers including information on chronic comorbidities from the Special Reimbursement Register (1972-2015), hospital admissions with diagnosis from the Hospital Discharge Register (1972-2015) and reimbursed prescription drug dispensing from the Prescription Register (1995-2015).

\section{Study design}

The study sample included all persons with and without $\mathrm{AD}$ with diabetes 5 years before the date of $\mathrm{AD}$ diagnosis (index date). Persons were considered to have diabetes if they were eligible for special reimbursement of diabetes drugs by the SII and/or if they had been dispensed reimbursed diabetes drugs according to Anatomical Therapeutic Chemical (ATC) classification [13]. The date for diabetes diagnosis was defined either as the date of entitlement for reimbursement, or the date of first purchase of diabetes drug, whichever occurred first.

Use of diabetes drugs were modelled from drug dispensings recorded in the Prescription Register with validated 'Prescriptions to drug use periods' (PRE2DUP) model [14]. This model considers regularity of drug dispensing, stockpiling of drugs and possible hospital/ institutional care when drugs are provided by the caring unit. Previous research has demonstrated good agreement between PRE2DUP modelled drug use and self-reported use of insulin and oral diabetes drugs [15]. Diabetes drugs were defined as insulins, metformin, sulfonylureas and other diabetes drugs including sulfonamides, alpha glucosidase inhibitors, thiazolidinediones, dipeptidyl peptidase 4 inhibitors (DPP-4), glucagon-like peptide-1 analogues (GPL-1), sodium-glucose co-transporter 2 inhibitors (SGLT2), and repaglinide, nateglinide, pramlintide, benfluorex and mitiglinide (Supplementary Table 1). The prevalence of diabetes drug use was defined in three-month evaluation periods from 5 years before until 5 years after the index date. Drugs used in public nursing homes and hospitals are not recorded in the Prescription Register. Therefore, only persons who were alive and not hospitalized nor a nursing home resident for more than 30 days of a specific evaluation period were included. Follow-up ended on the date of death, 5 years after index date, or at the end of data linkage (31 Dec 2015), whichever occurred first. In addition, persons in the non-AD cohort were censored from the study at their $\mathrm{AD}$ diagnosis date if they received $\mathrm{AD}$ diagnosis during the follow-up.

Under Finnish legislation, ethics committee approval was not required because no participants were contacted and data were de-identified by the data custodians prior to analyses by the researchers.

\section{Statistical analysis}

Characteristics of persons with $\mathrm{AD}$ and matched comparison persons without $\mathrm{AD}$ were presented using frequencies and percentages or means and standard deviations (SD). Categorical variables were compared by the Chi-square test and continuous variables by the T-test. The prevalence of diabetes drug use in each observation 
period were presented with $95 \%$ confidence intervals. All analyses were conducted using SPSS software (IBM SPSS Statistics Version 25) for Windows.

\section{Results}

There were 8418 persons with $\mathrm{AD}$ and 6666 persons without $\mathrm{AD}$ who had diabetes 5 years before the index date included in the study. The mean age was similar in both groups (79.9years and 80.7 years for persons with and without $\mathrm{AD}$, respectively) and the majority of persons were women in both cohorts $(59.2 \%$ and 63.0 in AD and non-AD cohorts) (Supplementary Table 2).

Nearly all persons with diabetes $(94.6 \%$ of the ADcohort and $94.0 \%$ of non-AD cohort) used at least one diabetes drug on the index date (Supplementary Table 2). Metformin was the most prevalent diabetes drug (60.9 and $59.1 \%$ in AD- and non-AD cohorts, respectively) followed by sulfonylureas and insulin. There were no differences in the prevalence of diabetes drug use between persons with and without $\mathrm{AD}$ prior to the index date (Fig. 1). The prevalence of diabetes drug use started to decline continuously 1 year after $\mathrm{AD}$ diagnosis until the end of follow-up in persons with $\mathrm{AD}$. In contrast, the prevalence of diabetes drug use remained constant in the comparison persons without $\mathrm{AD}$. At the end of the follow-up the prevalence of diabetes drug use was higher in people without $\mathrm{AD} 91.17 \%(95 \% \mathrm{Cl} 91.26-93.10 \mathrm{com}-$ pared to those with AD 85.33\% (95\% Cl 84.33-86.62).

The proportion of persons using insulin increased throughout the 10-year follow-up in both cohorts (from 26.2 to $51.8 \%$ and from 27.9 to $55.6 \%$ in cohorts without and with $\mathrm{AD}$, respectively) (Fig. 2A). The prevalence of metformin use decreased in both the $\mathrm{AD}$ and
non-AD cohorts, although this decrease was more pronounced in the AD cohort (Fig. 2B). The use of sulfonylureas decreased in both cohorts throughout the 10-year follow-up, but this decrease was also more pronounced in the AD cohort (Fig. 2C). Use of other diabetes drugs (including sulfonamides, alpha glucosidase inhibitors, thiazolidinediones and DPP-4 inhibitors, GLP-1 analogues, SGLT2 inhibitors) was rare (1.9\% in persons with $\mathrm{AD}, 2.2 \%$ in persons without $\mathrm{AD}$ ) at the beginning of the follow-up but increased over 10-fold until the end of the follow-up (26.6\% in $\mathrm{AD}$ and $31.5 \%$ in non-AD cohort, respectively) (Fig. 2D). The increase was primarily attributable to increased use of DPP-4 inhibitors, as the use of other diabetes drugs was minimal (data not shown). After the index date the increase in use was lower among people with $\mathrm{AD}$.

Use of short- and particularly long-acting insulins increased throughout the 10-year period in both cohorts (Fig. 3). The increase of long-acting insulins was more pronounced in persons with $\mathrm{AD}$ at the year of $\mathrm{AD}$ diagnosis and remained higher than in persons without $A D$ throughout the follow-up. Use of intermediate-acting insulin decreased over the follow-up and this decrease was more evident after index date among persons with AD. Use of mixed insulins was relatively stable in both cohorts over the follow-up.

\section{Discussion}

To our knowledge, this is the first study to report changes in the use of diabetes drugs in persons with and without AD. Diabetes drug use decreased one year after diagnosis in persons with AD compared to those without $\mathrm{AD}$. This finding is in accordance with a previous

Any diabetes drug

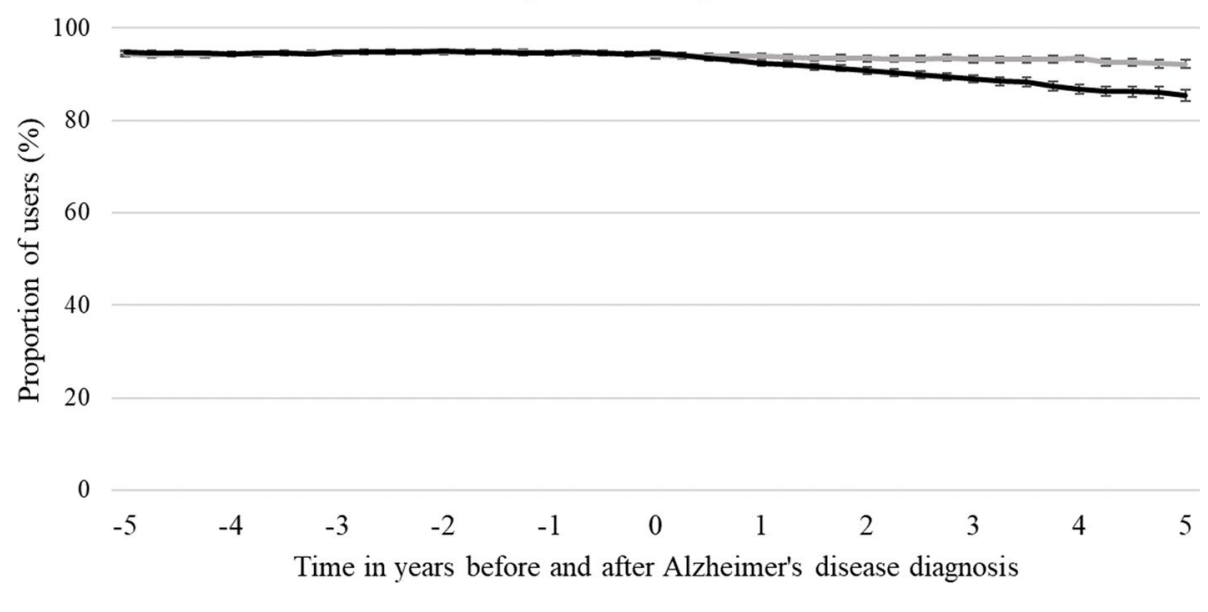

Users without $\mathrm{AD} \quad$-Users with $\mathrm{AD}$

Fig. 1 Prevalence of any diabetes drug use in persons with and without Alzheimer's disease (AD) with 95\% confidence intervals 


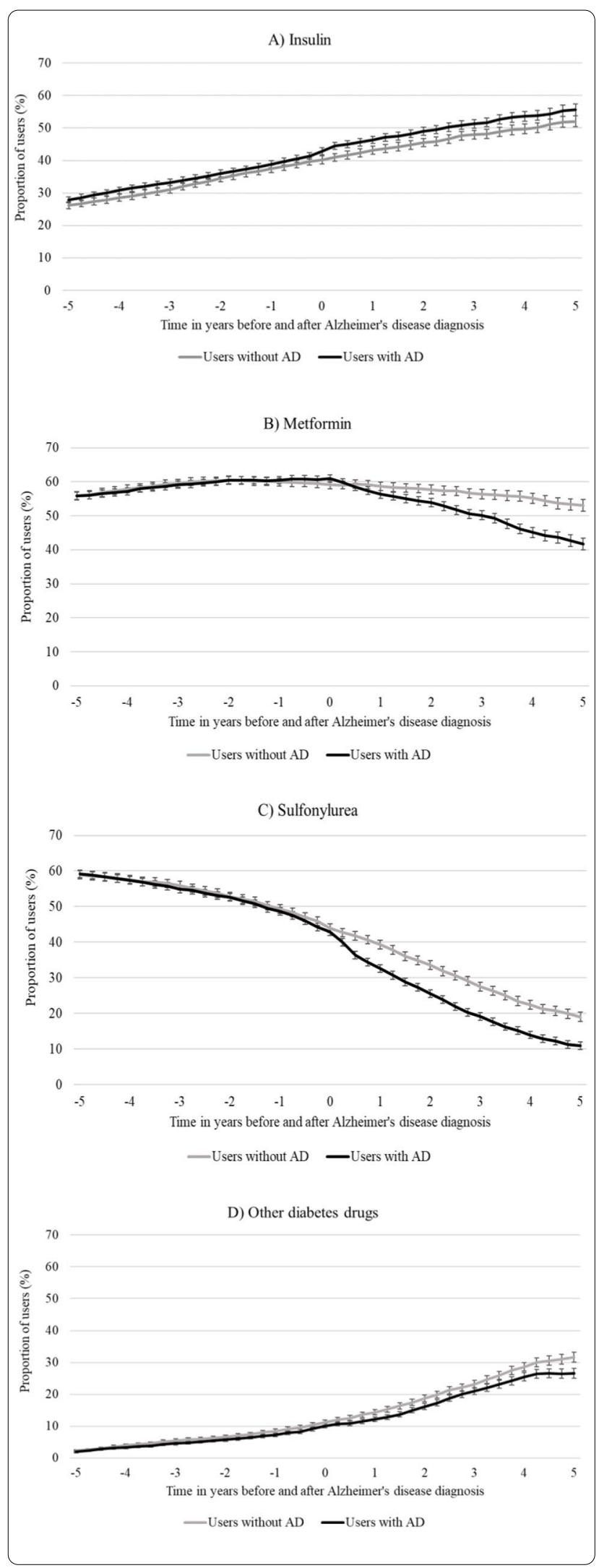

Fig. 2 Prevalence of use of a) insulin, b) metformin, c) sulfonylureas and d) other diabetes drugs in the cohort of persons without and with Alzheimer's disease (AD) with 95\% intervals

United States study on changes on drug utilization among people with diabetes following $\mathrm{AD}$ diagnosis [10]. The decrease in diabetes drug use may be partly attributable to unintentional weight loss and a corresponding reduction in the need for diabetes drugs after $\mathrm{AD}$ diagnosis [16, 17]. The decrease might also reflect recognition that tight glycemic control in vulnerable older people can lead to hypoglycemia [18], increased risk of falls and related fractures, micro- and macrovascular complications or even death [19]. In addition, weight loss is related to frailty which has been demonstrated to increase the risk of hypoglycemia in persons with dementia [20] Severe hypoglycemia is more common in people with diabetes and dementia compared to those without dementia [21]. Therefore, optimizing diabetes treatment regimens according to each individual's glycemic goals is encouraged in persons with cognitive impairment [18]. This approach is consistent with a focus on maintaining quality of life through avoiding hypoglycemia [7]. Our results are in contrast to a recent Australian study that reported clinicians appeared to prescribe more conservatively for people with diabetes who were older and frailer, but not for people with dementia [22].

In our study, metformin was the most frequently used diabetes drug at the index date among people with and without AD. Metformin has a favorable benefit-to-risk ratio although should be used with caution in renal impairment due to the risk of lactic acidosis [23, 24]. However, with appropriate dose adjustment metformin has been shown to be largely safe and effective in people with chronic kidney disease [25]. This is in line with guidelines recommending metformin as the first-line agent for type 2 diabetes [26]. This likely explains the frequent use of metformin in both cohorts. While these findings were consistent with international findings, older age has also been associated with higher odds of initiating non-metformin treatment [27].

Sulfonylureas have been associated with an increased risk of severe and long-lasting hypoglycemia, which may outweigh their benefits among older people [28, 29]. Furthermore, their beneficial effects may decrease after 2 years of use [26]. Introduction of newer and safer diabetes drugs is likely to explain the decreased use of sulfonylureas over time in both cohorts. The shift away from sulfonylureas may also reflect an international transition toward newer diabetes treatments such as SGLT-2 inhibitors, DPP-4 inhibitors and GLP-1 agonists [30]. 


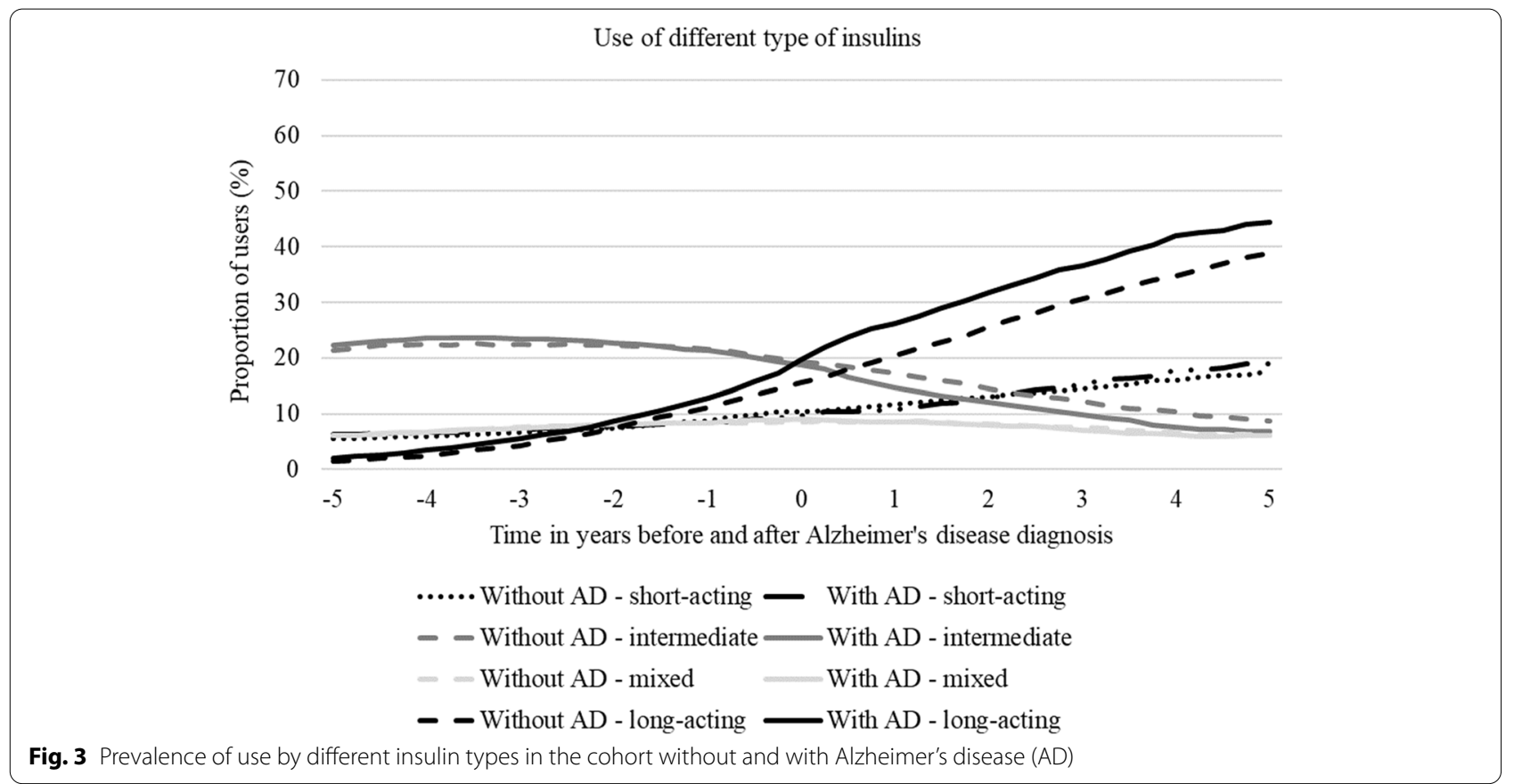

The use of long-acting insulin increased throughout the follow-up in people with and without AD. These changes were consistent with recommendations for insulin selection in older people with diabetes and cognitive impairment. Basal insulin is generally considered the insulin of choice due to the less frequent administration and reduced risk of adverse events $[7,31]$.

DPP-4 inhibitors were the most frequently used newer diabetes drugs whereas use of SGLT-2 inhibitors and thiazolidinediones was minimal. DPP-4 inhibitors have a favorable safety profile among older persons although caution is needed in patients with heart failure, and dose reduction is needed in renal impairment [27]. As these newer diabetes drugs became available during the follow-up, their prevalence was low at the beginning of the follow-up.

The main strength of the study was the large national population-based cohort of community-dwelling persons with a clinically verified diagnosis of AD. Data linkage enabled us to study drug use over a 10-year follow-up and avoid recall and selection biases. However, the Finnish Prescription Register did not include information about drug use during periods persons were in hospitals or public nursing homes. Therefore, we censored persons from the observation period if they stayed in hospitals or nursing homes for more than 30 days during each specific 3-month period. Consequently, our findings are only generalizable to community-dwelling persons. In addition, the Special Reimbursement Register lacked information about the type of diabetes. The registers also lacked the information on the severity of $\mathrm{AD}$ and clinical information on short- and long-term levels of blood glucose, glycosylated hemoglobin levels and other clinical factors that were likely to have affected the decision to prescribe and discontinue diabetes drugs. Further research is needed on the optimal management of diabetes in persons with dementia and to develop safe and feasible treatment regimens for this vulnerable population. This includes research into different diabetes treatment deintensification strategies in people with dementia.

\section{Conclusion and implications}

The prevalence of diabetes drug use decreases 1 year following diagnosis of $\mathrm{AD}$ compared to matched people not diagnosed with AD. The decrease in the overall use of diabetes drugs and increase in long-acting insulins after AD diagnosis likely reflects clinicians' and patients' attempts to avoid hypoglycemia in this vulnerable group of older persons.

\section{Abbreviations}

AD: Alzheimer's Disease; ATC: Anatomical Therapeutic Chemical; DPP-4: Dipeptidyl peptidase 4 inhibitors, DSM-IV: Diagnostic and Statistical Manual of Mental Disorders; GLP-1: Glucagon-like peptide-1 analogues; MEDALZ: Medication Use and Alzheimer's disease; NINCDS-ADRDA: National Institute of Neurological and Communicative Disorders and Stroke and the Alzheimer's Disease and Related Disorders Association; SD: Standard Deviation; SGLT2: Sodium-glucose co-transporter 2 inhibitors; SII: Social Insurance Institution. 


\section{Supplementary Information}

The online version contains supplementary material available at https://doi. org/10.1186/s12877-021-02694-w.

Additional file 1.

Additional file 2 .

\section{Acknowledgements}

Not applicable.

\section{Authors' contributions}

CLR: execution of research project, execution and review of statistical analysis, writing of the first draft, review and critique of manuscript. MK: research project conception, statistical analysis design and execution, review and critique, review and critique of manuscript. PL, HT, AT, JSB: review and critique of manuscript. AMT: research project conception, statistical analysis design, review and critique of manuscript. SH: research project conception and organization, statistical analysis design, review and critique of manuscript. The authors read and approved the final manuscript.

\section{Funding}

None.

\section{Availability of data and materials}

Restrictions apply to the availability of the data that support the findings of this study, so they are not publicly available. Data are available from the authors upon reasonable request and if appropriate permission from the Finnish Social and Health Data Permit Authority Findata is sought and granted.

\section{Declarations}

\section{Ethics approval and consent to participate}

Register maintainers have approved the MEDALZ study plan. Data were pseudonymized before submission to the research team and study participants were not contacted. Therefore, according to Finnish legislation (including Personal Data Act 23/1999, Act on the Openness of Government Activities $621 / 1999$ and Act on the Secondary Use of Health and Social Data 552/2019 (and previous Act on the National Healthcare registers, not official English translation as this is not available 556/1989) the study has been granted an exemption from requiring ethics approval or informed consent.

\section{Consent for publication}

\section{Not applicable.}

\section{Competing interests}

CLR, MK, PL, A-MT have no conflicts of interest. SH has got lecture fees from Astellas-Pharma. HT and AT have participated in research projects funded by grants from Janssen-Cilag and Eli Lilly to their employing institution. HT reports personal fees from Janssen-Cilag. JSB has received grant funding from the Australian National Health and Medical Research Council (NHMRC), Medical Research Future Fund (MRFF), Victorian Government Department of Health and Human Services (DHHS), Dementia Australia Research Foundation (DARF), Dementia Centre for Research Collaboration (DCRC), Yulgilbar Foundation, Pharmaceutical Society of Australia (PSA), GlaxoSmithKline Supported Studies Programme, and several aged care provider organizations over the past three years all unrelated to the present work. All grants and consulting funds were paid to the employing institution. JSB is supported by a NHMRC Boosting Dementia Leadership Fellowship.

\section{Author details}

${ }^{1}$ School of Medicine, University of Eastern Finland, Kuopio, Finland. ${ }^{2}$ Kuopio Research Centre of Geriatric Care, University of Eastern Finland, Kuopio, Finland. ${ }^{3}$ School of Pharmacy, University of Eastern Finland, PO Box 1627, 70210 Kuopio, Finland. ${ }^{4}$ Centre for Medicine Use and Safety, Faculty of Pharmacy and Pharmaceutical Sciences, Monash University, Parkville, Victoria, Australia. ${ }^{5}$ Department of Clinical Neuroscience, Karolinska Institutet, Stockholm,
Sweden. ${ }^{6}$ Department of Forensic Psychiatry, Niuvanniemi Hospital, University of Eastern Finland, Kuopio, Finland.

Received: 8 June 2021 Accepted: 29 November 2021

Published online: 15 December 2021

\section{References}

1. Lindeboom J, Weinstein H. Neuropsychology of cognitive ageing, minimal cognitive impairment, Alzheimer's disease, and vascular cognitive impairment. Eur J Pharmacol. 2004;490:83-6. https://doi.org/10.1016/j. ejphar.2004.02.046.

2. https://www.who.int/health-topics/diabetes\#tab=tab_1 Accessed 11 Nov 2021.

3. International Diabetes federation (2019) https://diabetesatlas.org/ idfawp/resource-files/2019/07/IDF_diabetes_atlas_ninth_edition_en.pdf Accessed 11 November 2021.

4. Centers of Disease Control and Prevention: Prevalence of both diagnosed and undiagnosed diabetes. 2020 https://www.cdc.gov/diabetes/pdfs/ data/statistics/national-diabetes-statistics-report.pdf

5. Tolppanen A, Lavikainen P, Solomon A, Kivipelto M, Uusitupa M, Soininen $\mathrm{H}$, et al. History of medically treated diabetes and risk of Alzheimer disease in a nationwide case-control study. Diabetes Care. 2013:36:2015-9. https://doi.org/10.2337/dc12-1287.

6. World Health Organization: Dementia. 2020, 2020 https://www.who.int/ news-room/fact-sheets/detail/dementia.

7. American Diabetes Association: 12. Older adults: standards of medical care in diabetes-2020. Diabetes Care. 2020;43(Suppl 1):S152-62. https:// doi.org/10.2337/dc20-S012

8. Biessels GJ, Whitmer RA: Cognitive dysfunction in diabetes: how to implement emerging guidelines. Diabetologia. 2020;63:3-9. https://doi.org/10. 1007/s00125-019-04977-9.

9. Stasinopoulos J, Bell JS, Manski-Nankervis JA, Hogan M, Jenkin P, Sluggett JK. Medication management of type 2 diabetes in residential aged care. Aust J Gen Pract. 2018;47(10):675-81. https://doi.org/10.31128/ AJGP-05-18-4582

10. Sarkar U, Lyles C, Steinman M, Huang ES, Moffet HH, Whitmer RA, et al. Changes in medication use after dementia diagnosis in an observational cohort of individuals with diabetes mellitus. J Am Geriatr Soc 2017;65:77-82. https://doi.org/10.1111/jgs.14429.

11. Tolppanen A, Taipale H, Koponen M, Lavikainen P, Tanskanen A, Tiihonen J, et al. Cohort profile: the Finnish medication and Alzheimer's disease (MEDALZ) study. BMJ Open. 2016;6(7):e012100. https://doi.org/10.1136/ bmjopen-2016-012100.

12. McKhann G, Drachman D, Folstein M, Katzman R, Price D, Stadlan EM Clinical diagnosis of Alzheimer's disease. Clinical diagnosis of Alzheimer's disease: report of the NINCDS-ADRDA Work Group under the auspices of Department of Health and Human Services Task Force on Alzheimer's Disease. Neurology. 1984;34:939. https://doi.org/10.1212/wnl.34.7.939.

13. WHO Collaborating Centre for Drug Statistics Methodology: Guidelines for ATC classification and DDD assignment. 2020 https://www.whocc.no/ atc_ddd_index_and_guidelines/guidelines/

14. Tanskanen A, Taipale H, Koponen M, Tolppanen A, Hartikainen S, Ahonen $\mathrm{R}$, et al. From prescription drug purchases to drug use periods - a second generation method (PRE2DUP). BMC Med Inform Decis Mak. 2015;15:21. https://doi.org/10.1186/s12911-015-0140-z.

15. Taipale H, Tanskanen A, Koponen M, Tolppanen A, Tiihonen J, Hartikainen S. Agreement between PRE2DUP register data modeling method and comprehensive drug use interview among older persons. Clin Epidemiol. 2016:8(363-371). https://doi.org/10.2147/CLEP.S116160 eCollection 2016.

16. Gillette-Guyonnet S, Nourhashémi F, Andrieu S, de Glisezinski I, Ousset PJ, Rivière D, et al. Weight loss in Alzheimer disease. Am J Clin Nutr. 2000;71:637S-42S. https://doi.org/10.1093/ajcn/71.2.637s.

17. Abdelhafiz AH, Chakravorty P, Gupta S, Haque A, Sinclair AJ. Can hypoglycaemic medications be withdrawn in older people with type 2 diabetes? Int J Clin Pract. 2014;68:790-2. https://doi.org/10.1111/ijcp.12455.

18. Tseng C, Soroka O, Maney M, Aron DC, Pogach LM. Assessing potential glycemic overtreatment in persons at hypoglycemic risk. JAMA. Intern Med. 2014;174(2):259-68. https://doi.org/10.1001/jamainternmed.2013. 12963. 
19. Mattishent K, Loke YK. Meta-analysis: Association between hypoglycaemia and serious adverse events in older patients. J Diabetes Complicat. 2016;30:811-8. https://doi.org/10.1016/j.jdiacomp.2016.03.018.

20. Abdelhafiz AH, McNicholas W, Sinclair AJ. Hypoglycemia, frailty and dementia in older people with diabetes: reciprocal relations and clinical implications. J Diabetes Complicat. 2016;30(8):1548-54. https://doi.org/ 10.1016/j.jdiacomp.2016.07.027.

21. Prinz N, Stingl J, Dapp A, Denkinger MD, Fasching P, Jehle PM, et al. DPV initiative. High rate of hypoglycemia in 6770 type 2 diabetes patients with comorbid dementia: a multicenter cohort study on 215,932 patients from the German/Austrian diabetes registry. Diabetes Res Clin Pract. 2016;112:73-81. https://doi.org/10.1016/j.diabres.2015.10.026.

22. Wood SJ, Bell JS, Magliano DJ, Fanning L, Cesari M, Keen CS, et al. Impact of age, frailty, and dementia on the types of type 2 diabetes therapies prescribed at hospital discharge 2012-2016. J Frailty Aging. 2021;10(4):343-9. https://doi.org/10.14283/jfa.2021.6.

23. Schlender L, Martinez YV, Adeniji C, Reeves D, Faller B, Sommerauer C, et al. Efficacy and safety of metformin in the management of type 2 diabetes mellitus in older adults: a systematic review for the development of recommendations to reduce potentially inappropriate prescribing. BMC Geriatr. 2017;17(Suppl 1):227. https://doi.org/10.1186/s12877-017-0574-5.

24. Abdelhafiz AH. Diabetic kidney disease in older people with type 2 diabetes mellitus: improving prevention and treatment options. Drugs Aging. 2020;37(8):567-84. https://doi.org/10.1007/s40266-020-00773-y.

25. Lalau J, Kajbaf F, Bennis Y, Hurtel-Lemaire A, Belpaire F, De Broe ME. Metformin treatment in patients with type 2 diabetes and chronic kidney disease stages 3A, 3B, or 4. Diabetes Care. 2018;41(3):547-53. https://doi. org/10.2337/dc17-2231

26. Working group set up by the Finnish Medical Society Duodecim, the Finnish Society of Internal Medicine and Finnish Diabetes Association: Type 2 diabetes current care guidelines. 2020 https://www.kaypahoito.fi/ hoi50056. Published 18th May 2020.

27. Wood SJ, Magliano DJ, Bell JS, Shaw JE, Keen CS, Ilomäki J. Pharmacological treatment initiation for type 2 diabetes in Australia: are the guidelines being followed? Diabet Med. 2020;37(8):1367-73. https://doi.org/10. 1111/dme.14149.

28. Yu O, Azoulay L, Yin H, Filion KB, Suissa S. Sulfonylureas as initial treatment for type 2 diabetes and the risk of severe hypoglycemia. Am J Med. 2018;131(3):317.e11-22. https://doi.org/10.1016/j.amjmed.2017.09.044.

29. American Geriatrics Society beers criteria expert panel: American geriatrics society 2019 updated AGS beers criteria ${ }^{\circledR}$ for potentially inappropriate medication use in older adults. J Am Geriatr Soc. 2019;67:674-94. https://doi.org/10.1111/jgs.15767.

30. Chin KL, Hidayat FM, Ofori-Asenso R, Ilomäki J, Bell JS, Zoungas S, et al. Trends in the dispensing and costs of glucose-lowering medications among older Australians: Findings from National Claims Data. Drugs Aging. 2020;37:393-8. https://doi.org/10.1007/s40266-020-00759-w.

31. Schernthaner G, Schernthaner-Reiter MH. Diabetes in the older patient: heterogeneity requires individualisation of therapeutic strategies. Diabetologia. 2018;61:1503-16. https://doi.org/10.1007/s00125-018-4547-9.

\section{Publisher's Note}

Springer Nature remains neutral with regard to jurisdictional claims in published maps and institutional affiliations.

Ready to submit your research? Choose BMC and benefit from:

- fast, convenient online submission

- thorough peer review by experienced researchers in your field

- rapid publication on acceptance

- support for research data, including large and complex data types

- gold Open Access which fosters wider collaboration and increased citations

- maximum visibility for your research: over $100 \mathrm{M}$ website views per year

At BMC, research is always in progress.

Learn more biomedcentral.com/submissions 